\title{
COMBUSTION ANALYSIS BY IMAGE PROCESSING OF PREMIXED FLAMES
}

\author{
Gaetano Baldini \\ ENEL-CESI \\ via Rubattino, 54 \\ 20134 Milano
}

\author{
Paola Campadelli*†, Raffaella Lanzarotti \\ Università degli Studi di Milano \\ Dipartimento di Scienze dell'Informazione \\ Via Comelico, 39, I-20135 Milan, Italy
}

\begin{abstract}
We describe a three-step algorithm for the analysis of color images of flames, with the objective of analyzing the combustion process and its control parameters indirectly. The algorithm first extracts the interesting regions, applying a clustering method in the RGB color space, identifying and eliminating irrelevant regions and applying morphological operators. Then it calculates different geometrical parameters for each image. In the final step, it produces the temporal trend and the frequency spectrum for each parameter. The spectra are considered useful for the validation of a theoretical model about the instability phenomenon generated by premixed flames.
\end{abstract}

Keywords: image processing, premixed flames, combustion instability.

\section{INTRODUCTION}

The objective of increasing combustor's performance, and, at the same time, reducing pollution's (NO) emission in gas turbine combustors, has brought to the development of new combustion systems which use premixed flames [1].

A disadvantage of these systems is that premixed combustors are subjected to combustion instability [2][3].

This phenomenon is extremely dangerous for the fireboxes' integrity: pressure oscillations produce vibrations that can cause structural yielding and high level of acoustic noise; therefore the problem to solve is to lower the combustion instability in gas turbine combustors when premixed flames are used.

At the present, numerous gas turbine combustor builders' laboratories and many research institutes are experimenting active combustion control in order to eliminate the instability. This technique is supposed to attain its purpose by modulating certain variables, in particular fuel flow modulation looks the most promising proposal. With this object in view, at the ENEL-CESI Research Laboratory in Segrate (Mi) an

\footnotetext{
*Email: campadelli@dsi.unimi.it

† Thanks to Progetto MURST "Modelli di calcolo innovativi: metodi sintattici e combinatori' for funding.
}

experimental apparatus, based on a 'Rijke tube' [Fig. 1], has been built.

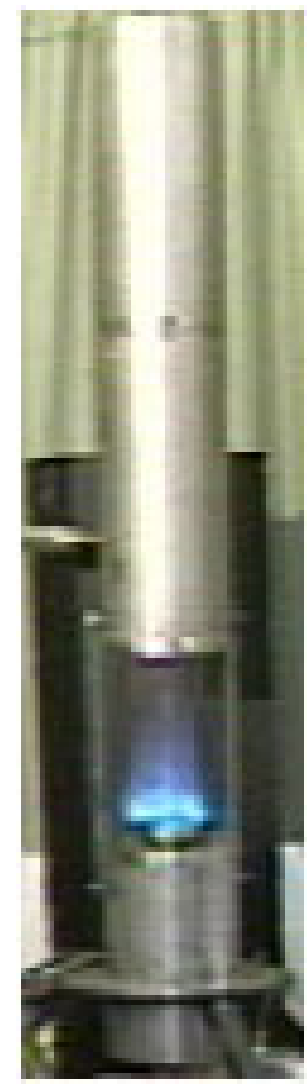

Fig. 1. Rijke tube.

It is therefore possible to generate the instability phenomenon due to premixed flames and gather experimental data (fuel flow, pressure, and temperature in different position in the tube). On these data a theoretical model based on the set of the non linear fluid-dynamic Navier-Stokes equations, has been developed[4]; the model predicts that the lowest and warmest region of the flame changes periodically with a main frequency at around $150 \mathrm{~Hz}$. This is also the typical frequency of the acoustic pressure inside 
the tube.

Starting from the hypothesis that there is a tight link between the control parameters and the flame shape, we developed an algorithm, based on off-line image processing, which computes the temporal behavior of some geometrical parameters used to describe the flame shape. Transforming these temporal signals into frequency domain we can compare our results with the theoretical hypothesis.

\section{PROPOSED METHOD FOR THE ANALYSIS OF THE IMAGES}

The procedure we have implemented [5] is divided into three principal steps: identification of interesting areas, calculation of geometrical parameters and definition of temporal trends and of frequency spectra.

These steps are applied on a sequence of 500 images (corresponding to one second of data acquisition).

\subsection{Identification of the interesting areas}

Image quality is very low, because of the necessity of acquiring them with high frequency (500 frames/s). This ensures us to catch phenomena up to $250 \mathrm{~Hz}$. Not the whole flame is visible but just the lowest and hottest part which is relevant for our research.

To lower the computational cost, we first eliminated most of the background by selecting smaller images from the original ones. This step requires that the user draws a rectangle on the first image only [Fig. 2]; the same area will be selected for all the other images automatically.

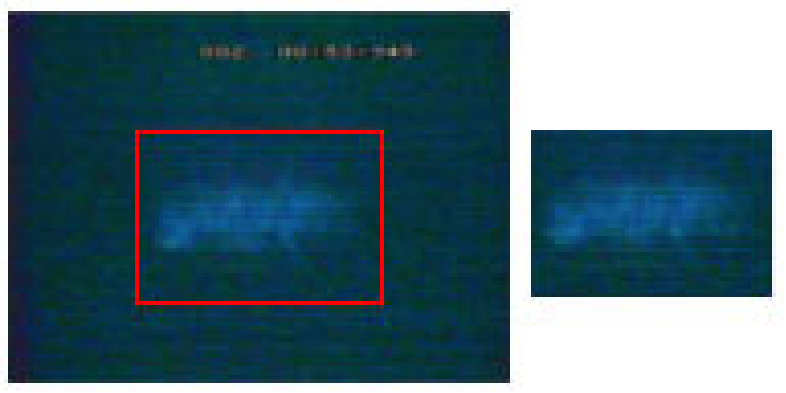

Fig. 2. Result of the selection.

What we want to identify in each image [Fig. 3.1] is the blue 'cloud' in the centre of the picture, that is the hottest and most significant part of the flame in instability conditions. We experimented with some standard techniques of image segmentation; in particular, we implemented and applied methods of edge detection (Sobel, Marr-Hildreth, Canny and their variations) and clustering in color space as a preliminary segmentation step. The clustering technique has given better results; of the algorithms considered
(Isodata[6], Arbib[7], deterministic annealing[8], fuzzy Cmeans[9]) we chose Arbib's, for its efficiency and simplicity of implementation. The clustering algorithm is applied in the RGB color space, having set the number of clusters to identify at three: two centroids refer to the background, and one refers to the interesting area [Fig. 3.2]. In order to obtain a binary image we have to eliminate the background; to achieve this objective we observed that, in each image, the cloud is always in the centre; we used this information to find its color: it is the color of most pixels of the $3 \times 3$ square in the centre of the image. Afterwards, we put at 0 all the pixels with a color different from the one selected and at 1 the others, obtaining a binary image [Fig. 3.3]. The binary image just obtained, shows two defects: a high number of thin 'filaments' and little holes inside the region. These defects have been eliminated respectively applying the opening and the closing operators[6] with an 8-metric disk of radius 3 as structuring element. The result is a more homogeneous region.

We then applied an algorithm that labels the connected components and maintained only the biggest one corresponding to the region we wanted to isolate [Fig. 3.4].

The last phase in this first step is the identification of the boundary of the region obtained [Fig. 3.5].

\subsection{Calculation of the geometrical parameters}

To describe the regions obtained, we used the following characteristics[6]: Area, Perimeter, Compactness, Centroid, Bounding rectangle, Axis length of the best fitting ellipse and its Extreme points, Ellipse eccentricity.

The extraction of these numerical parameters from the images, allows to represent the flame shape by a few parameters. In figure 3.6 some of them are represented.

\subsection{Definition of temporal trends and of their frequency spectra}

The values of each morphological parameter, calculated for each image, are treated as samples; observing their trends the same periodicity can be noticed.

In order to analyze the periodicity, the frequency spectrum for each temporal trend has been calculated by means of the DFT.

\section{RESULTS AND CONCLUSIONS}

Examining the spectra obtained we observed that geometrical parameters linked with the flame vertical (y) movement are more significant than the ones linked with the horizontal (x) movement. This result confirms the idea that the flame is continuously compressed and expanded by the air column over it. Among the measured geometrical parameters the $y$-coordinate of the centroid gives the most significant 


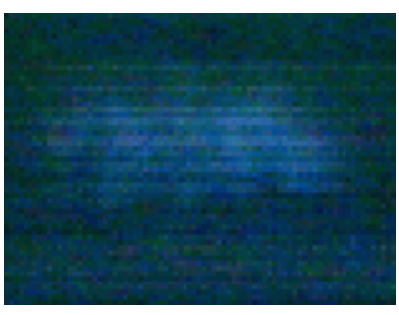

(1)

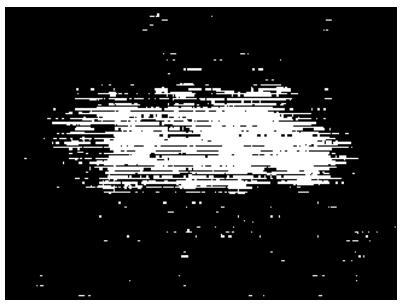

(3)

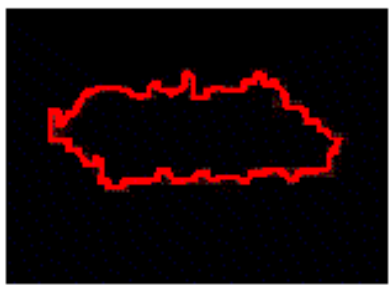

(5)

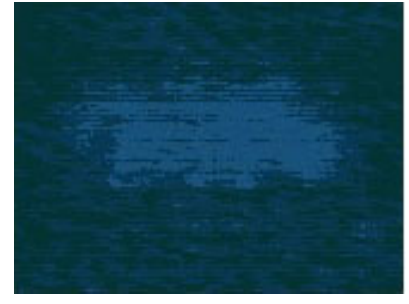

(2)

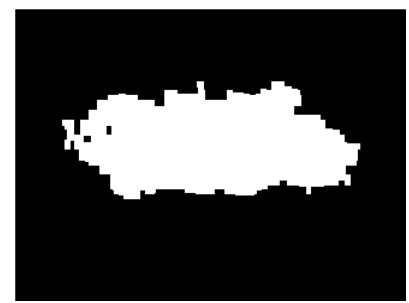

(4)

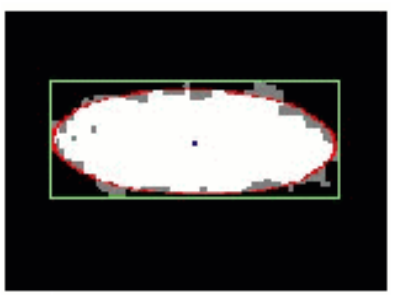

(6)
Fig. 3. Image processing applied

information, while the $\mathrm{x}$-coordinate of the extreme points gives the less significant. Figures 4 and 5 show a typical behavior of the most and less significant parameters. From the spectrum of the $y$-coordinate of the centroid, a peak at the frequency of $148 \mathrm{~Hz}$ can easily be recognized, confirming the theoretical hypothesis. Moreover there are two significant symmetric spectral lines at about 146 and $150 \mathrm{~Hz}$ which are due to the modulation induced on the acoustic field by the exhaust gas flow. The frequency of this modulation can be used to estimate the velocity of the exhaust gas.

The result obtained has been considered very useful by the modelers of the instability phenomena, because it gives them a further experimental tool to analyze the combustion and strengthens their theoretical hypothesis helping them in the tuning of some parameters of the model. Since the Rijke tube is an experimental device which well models the instability phenomena taking place in gas turbine power plants, the described algorithm could be conveniently used for studying the combustion instability in these plants. ${ }^{1}$

\footnotetext{
${ }^{1}$ The application has been developed using IDL. 5.0 of the RSI.
}

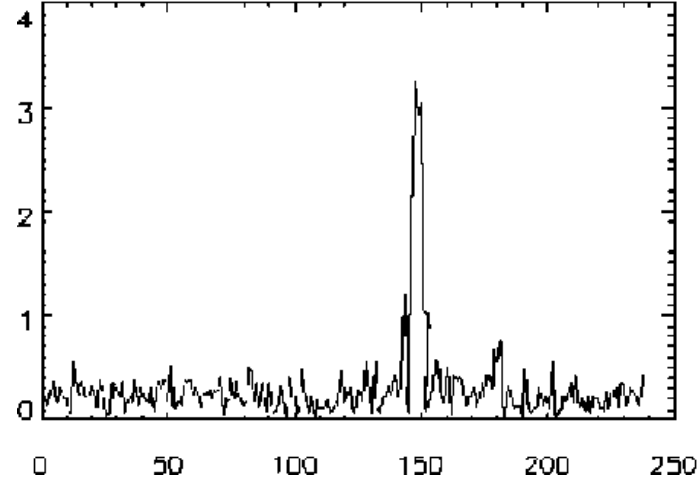

Fig. 4. Spectrum of the Y-coordinate of the centroid.

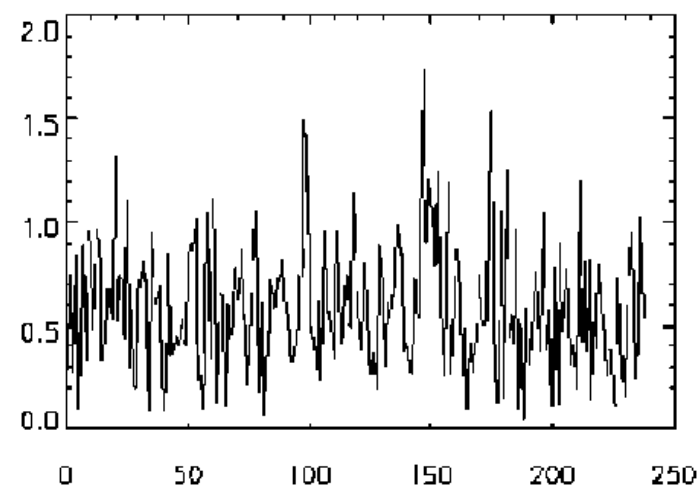

Fig. 5. Spectrum of the maximum $X$ in the best fitting ellipse.

\section{REFERENCES}

[1] J.B.Edwards, Combustion, Ann Arbor Science Publisher, 1974

[2] F.E.C.Culick, Non-linear growth and limiting amplitude of acoustic oscillations in combustion chambers, Combustion Science and Technology, vol. 3, pp. 1-16, 1971

[3] K. R. McManus, T. Poinsot, S. M. Candel, A Review of Active Control of Combustion instabilities, Prog. Energy Combust. Sci., vol. 19, pp. 1-29, 1993

[4] Baldini et alii., Modelling the Nonlinear Dynamics of Combustion Instabilities in Gas Turbines Towards Active Control, 14th IFAC World Congress,Bejing, China, 1999

[5] R. Lanzarotti, Metodi di elaborazione di immagini per l'individuazione di instabilità di combustione in bruciatori a fiamma premiscelata, M. Sc. Thesis, DSI, Dipartimento di Scienze delle informazioni, 1999 (In italian)

[6] R. M. Haralick e L.G. Shapiro, Computer and robot vision, Addison-Wesley publishing company, 1992 
[7] T. Uchiyama e M. A. Arbib, Color image segmentation using competitive learning, IEEE Trans. on Pattern Analysis and Machine Intelligence, vol. 16, n. 12, pp. 1197-1206, 1994
[8] K. Rose , E. Gurewitz, Constrained clustering as an optimization method, IEEE Trans. Pattern Anal. Machine Intell., vol. 15, n. 8, pp. 785-794, 1993

[9] J.C. Beztek and S.K. Pal, Fuzzy models for pattern recognition, publ. IEEE New-York, 1992 